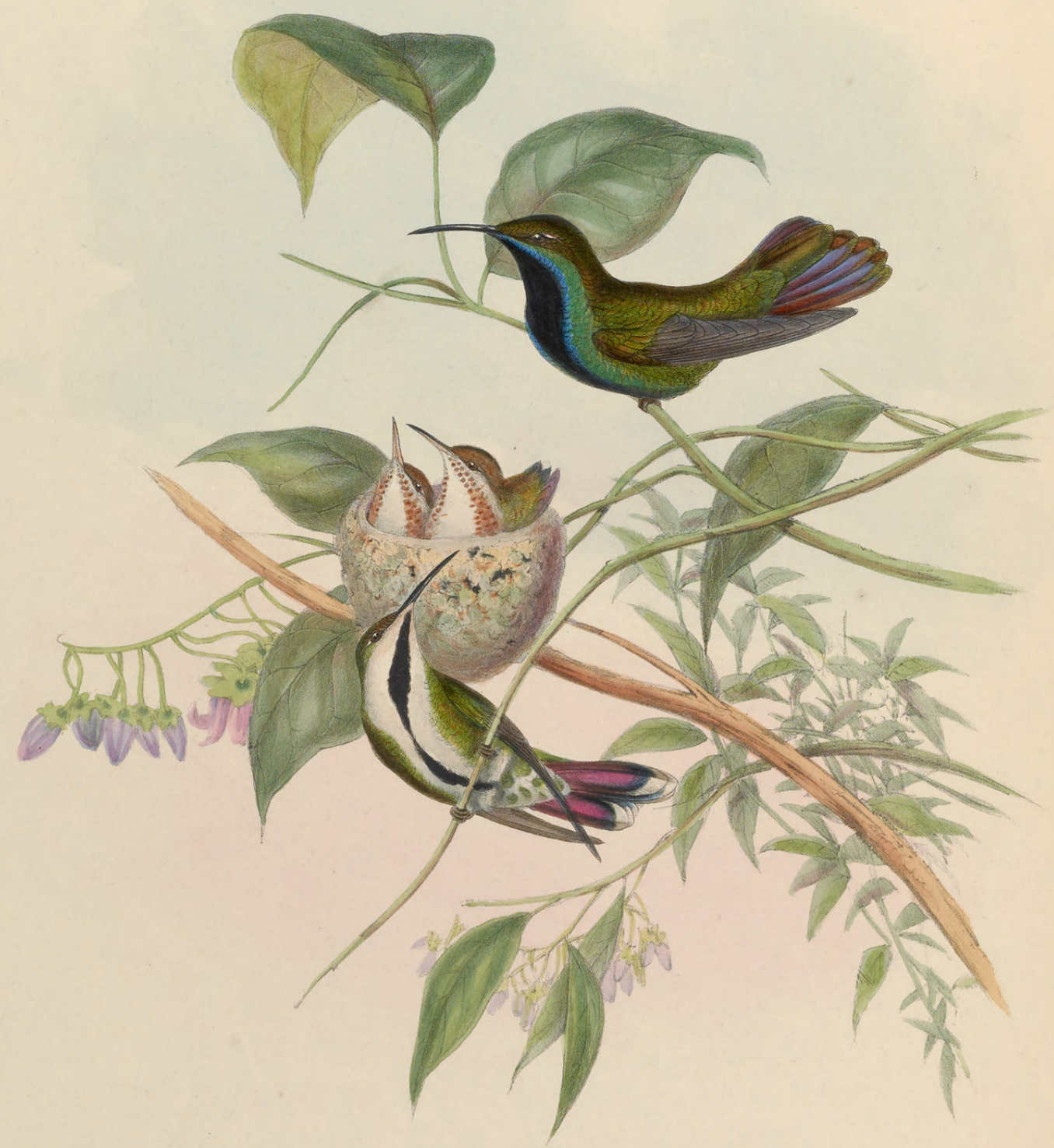




\title{
LAMPORNIS MANGO.
}

\author{
The Mango.
}

Trochilus Mango, Linn. Syst. Nat., tom. i. p. 191.-Ib. Gmel. Edit., tom. i. p. 491.-Shaw, Gen. Zool., vol. viii. p. 294.-Lath. Ind. Orn., vol. i. p. 307.-Less. Hist. Nat. des Col., p. 58. pls. 13, 13 bis, 14.-Vieill. Nouv. Dict. d'Hist. Nat., tom. vii. p. 353. -Ib. Ency. Méth. Orn., part ii. p. 557.-Drap. Dict. Class. d'Hist. Nat., tom. iv. p. 319.-Dumont, Dict. Sci. Nat., tom. x. p. 50.—Jard. Nat. Lib. Humming Birds, vol. ii. p. 100. pl. 20.-Swains. Birds of Brazil, pls. 27, 28.-Pr. Max. Beitr. zur Naturg. von Bras., B. iv. Div. i. p. 47.-Aud. Birds of Am., vol. ii. pl. clxxiv.Ib. Orn. Bio., vol. ii. p. 480.-Ib. Syn. Birds of Amer., p. 170.

Le Plastron noir, Buff. Hist. Nat. des Ois., tom. vi. p. 59.-Vieill. Ois. dor., tom. i. p. 20. pl. 7. Le Colibri du Mexique, Buff. Pl. Enl. 680. figs. 2, 3.

Trochilus violicauda, Bodd.

albus, Gmel. Edit. Linn. Syst. Nat., tom. i. p. 488.

nitidus, Lath. Ind. Orn., vol. i. p. 305.-Shaw, Gen. Zool., vol. viii. p. 301.

Le Colibri à queue violette, Buff. Hist. Nat. des Ois., vol. vi. p. 55.-Ib. Pl. Enl. 671. fig. 2.— Vieill. Ois. dor., tom. i. p. 27. pl. 11.

Trochilus punctulatus, Gmel. Edit. Linn. Syst. Nat., tom. i. p. 488.-Vieill. Ency. Méth. Orn., vol. ii. p. 652 ?-Lath. Ind. Orn., vol. i. p. 306.-Shaw, Gen. Zool., vol. viii p. 303.

Le Bec-fleurs à bande noir le long du corps, Azara, Voy. dans l'Amér. Mér., Sonn. Edit., vol. iv. p. 89. no. ccxcv.

Le Bec-fleurs bleu en dessous, Ib., p. 91. no. ccxcvi.

Le Bec-fleurs peint, Ib., p. 92. no. ccxcvin.

Polytmus punctulatus, Briss. Orn., tom. iii. p. 669.

Trochilus atricapillus, Vieill. 2nde Edit. du Nouv. Dict. d'Hist. Nat., tom. vii. p. 354.-Ib. Ency. Méth. Orn., part ii. p. 553.

fasciatus, Shaw, Gen. Zool., vol. viii. p. 303. - quadricolor, Vieill. Ency. Méth. Orn., part ii. p. 573.

Le Colibri à ventre piqueté, Vieill. Ois. dor., tom. i. p. 21. pl. 8.

Trochilus nigricollis, Vieill. 2nde Edit. du Nouv. Dict. d'Hist. Nat., tom. vii. p. 349 ?-Ib. Ency. Méth. Orn., pp. 2, 553?

Lampornis mango, Swains. Zool. Journ., vol. iii. p. 358.-Ib. Class. of Birds, vol. ii. p. 330.Bonap. Consp. Gen. Av., p. 71, Lampornis, sp. 1.-Ib. Rev. et Mag. de Zool. 1854, p. 250.

Polytmus mango, Gray and Mitch. Gen. of Birds, vol. i. p. 107, Polytmus, sp. 10.

Mango Humming Bird, Lath. Gen. Syn., vol. ii. p. 758.-Ib. Gen. Hist., vol. iv. p. 310._-Shaw, Gen. Zool., vol. viii. p. 294.

Violet-tailed Humming Bird, Lath. Gen. Syn., vol. ii. p. 754.-Shaw, Gen. Zool., vol. viii. p. 301.-Lath. Gen. Hist., vol. iv. p. 303.

Anthracothorax Mango, Reichenb. Aufz. der Colibris, p.11.-Ib.Troch. enumer., p.8. pl. Dccclx. figs. 4839-4841.

Purple-tailed Humming Bird, Shaw, Gen. Zool., vol. viii. p. 296.-Ib. Nat. Misc., pl. 333.

Spotted-necked Humming Bird, Lath. Gen. Syn., vol. ii. p. 755.

THIs familiar species of Humming Bird was not only known to Linnæus, but to Ray, Willoughby, Marcgrave, and most of the older authors, and is in fact one of those with which Europeans first became acquainted ; nevertheless, much confusion exists with regard to its synonymy, which I believe is principally owing to a nearly allied species, the Lampornis porphyrurus of Jamaica, having been confounded with it. This bird then it must be understood never goes to Jamaica, and to but few of the West India Islands; on the mainland, however, it enjoys a more extensive range than any other species. Audubon has included a 


\section{$2 \mathrm{BHL}$ Biodiversity Heritage Library}

Gould, John. 1853. "Lampornis mango, The Mango. [PI. 74]." A monograph of the Trochilidae, or family of humming-birds 2, https://doi.org/10.5962/p.316883.

View This Item Online: https://www.biodiversitylibrary.org/item/108332

DOI: https://doi.org/10.5962/p.316883

Permalink: https://www.biodiversitylibrary.org/partpdf/316883

\section{Holding Institution}

Smithsonian Libraries

\section{Sponsored by}

Smithsonian Institution Libraries

\section{Copyright \& Reuse}

Copyright Status: NOT_IN_COPYRIGHT

This document was created from content at the Biodiversity Heritage Library, the world's largest open access digital library for biodiversity literature and archives. Visit BHL at https://www.biodiversitylibrary.org. 